\title{
Identification of novel non-coding RNAs using profiles of short sequence reads from next generation sequencing data
}

\author{
Chol-Hee Jung ${ }^{1}$, Martin A Hansen ${ }^{1}$, Igor V Makunin ${ }^{1,2}$, Darren J Korbie' ${ }^{1}$, John S Mattick ${ }^{1 *}$
}

\begin{abstract}
Background: The increasing interest in small non-coding RNAs (ncRNAs) such as microRNAs (miRNAs), small interfering RNAs (siRNAs) and Piwi-interacting RNAs (piRNAs) and recent advances in sequencing technology have yielded large numbers of short (18-32 nt) RNA sequences from different organisms, some of which are derived from small nucleolar RNAs (snoRNAs) and transfer RNAs (tRNAs). We observed that these short ncRNAs frequently cover the entire length of annotated snoRNAs or tRNAs, which suggests that other loci specifying similar ncRNAs can be identified by clusters of short RNA sequences.

Results: We combined publicly available datasets of tens of millions of short RNA sequence tags from Drosophila melanogaster, and mapped them to the Drosophila genome. Approximately 6 million perfectly mapping sequence tags were then assembled into 521,302 tag-contigs (TCs) based on tag overlap. Most transposon-derived sequences, exons and annotated miRNAs, tRNAs and snoRNAs are detected by TCs, which show distinct patterns of length and tag-depth for different categories. The typical length and tag-depth of snoRNA-derived TCs was used to predict 7 previously unrecognized box H/ACA and 26 box C/D snoRNA candidates. We also identified one snRNA candidate and 86 loci with a high number of tags that are yet to be annotated, 7 of which have a particular 18mer motif and are located in introns of genes involved in development. A subset of new snoRNA candidates and putative ncRNA candidates was verified by Northern blot.

Conclusions: In this study, we have introduced a new approach to identify new members of known classes of ncRNAs based on the features of TCs corresponding to known ncRNAs. A large number of the identified TCs are yet to be examined experimentally suggesting that many more novel ncRNAs remain to be discovered.
\end{abstract}

\section{Background}

Following the discovery of microRNAs (miRNAs) and the RNA interference pathway in C. elegans [1], and the realisation that small RNAs are central to many aspects of plant and animal gene regulation, especially during development $[2,3]$, have led to the identification of thousands of miRNAs in many species through deep sequencing-based approaches [4-6]. Such approaches have also identified related small ncRNAs including small interfering RNAs (siRNAs) and Piwi-interacting RNAs (piRNAs) that are involved in RNA-silencing pathways in somatic and germline cells, respectively [7-10]. Moreover, recent advances in sequencing technology have

\footnotetext{
* Correspondence: j.mattick@imb.uq.edu.au

'Institute for Molecular Bioscience, University of Queensland, St Lucia QLD
} 4072, Australia increased the understanding of biogenesis of these ncRNAs through deep-sequencing of size-fractionated RNA fragments associated with particular proteins [8,9,11-20], and also increased the amount of available short RNA sequencing data dramatically.

Analysis of such data has shown that miRNA-sized short RNA fragments are commonly derived from other small RNAs, notably transfer RNAs (tRNAs) and small nucleolar RNAs (snoRNAs) [21,22]. Indeed it is now evident that almost all snoRNAs produce defined classes of small RNAs that have characteristic sizes, origins within the snoRNAs, and 5' nucleotide biases [22], some of which may function as miRNAs [23]. In analyzing such data we observed that many annotated snoRNAs and other ncRNAs are in fact covered with overlapping short RNA tags across their full length, although most are derived 
from particular locations within the ncRNAs. A similar observation was also employed recently for computational prediction of novel snoRNAs in the Arabidopsis genome [24]. The observation of precursor coverage suggested to us that the profiles of overlapping short RNA sequences might identify novel members of known ncRNA classes and perhaps putative novel species of ncRNAs. In this study we confirm this prediction by using assembled short RNA sequences to identify one new snRNA, 7 new box $\mathrm{H} /$ ACA and 26 new box C/D snoRNAs, as well as a number of novel ncRNAs.

\section{Results}

\section{Compilation of short RNA sequence reads into tag- contigs}

We obtained 10,846,433 sequence tags comprising 55,894,809 reads from 12 Gene Expression Omnibus (GEO) datasets (Table 1) derived from 90 experiments performed on Drosophila cell lines and tissues. Approximately 6 million tags were perfectly mapped to the $D$. melanogaster genome, excluding chrM (mitochondrial DNA), and chrU and chrUextra (which contain unassembled and un-mapped scaffolds). Each "tag" consists of one to many reads which reflects the number of times the tag was cloned and sequenced. For tags mapping to multiple locations on the genome, the number of reads of the given tag was arbitrarily distributed evenly to each mapping locus (see Methods). The tags were then assembled into 521,302 tag-contigs (TCs), comprised of contiguously overlapping (by 1 nt or more) tags present in the same strand orientation (Fig. 1) (see Methods). As a measure of expression level, each TC was assigned a tag-depth score based on the maximum number of overlapping reads covering any part of the locus (Fig. 1) (see Methods).

Table 1 Publicly available short RNA sequencing datasets on D. malanogaster

\begin{tabular}{llll}
\hline GEO accession & No. of tags & Mappable & References \\
\hline GSE10277 & 23252 & 12057 & {$[14]$} \\
\hline GSE10515 & 49878 & 12096 & {$[15]$} \\
\hline GSE10790 & 347861 & 30780 & {$[19]$} \\
\hline GSE10794 & 1387144 & 692422 & {$[16]$} \\
\hline GSE11019 & 255670 & 381508 & {$[9]$} \\
\hline GSE11086 & 1277025 & 1509771 & {$[13]$} \\
\hline GSE11624 & 6643474 & 3125323 & {$[12]$} \\
\hline GSE6734 & 32160 & 34362 & {$[8]$} \\
\hline GSE7448 & 753797 & 452471 & {$[17]$} \\
\hline GSE9138 & 13299 & 13294 & {$[20]$} \\
\hline GSE9389 & 59906 & 32472 & {$[18,9]$} \\
\hline GSE12527 & 2967 & 817 & {$[11]$} \\
\hline total & 10846433 & 6297373 & \\
\hline
\end{tabular}

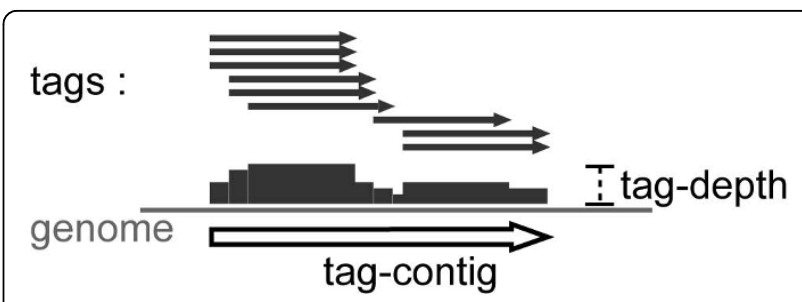

Figure 1 Compilation of a tag-contig. Contiguously overlapping tags (grey arrows) were assembled into a tag-contig (TC) (block arrow). The tag-depth is the maximum number of overlaying sequence reads per each base (grey blocks) within a given TC.

The 521,302 TCs, ranging from 12 nt to $2734 \mathrm{nt}$ in length and from 1 to 1767905 in tag-depth, occupy 14.6 Mbp (11.3\%) of Drosophila genome in total (excluding chrU, chrUextra and chrM). Several studies have identified siRNAs and piRNAs processed from transposons such as LINEs, DNAs and LTRs, and correspondingly there is considerable $\mathrm{TC}$ coverage over these repeat elements (Additional file 1, Table S1). Most exons (75\%) are also overlapped by TCs, possibly derived from messenger RNA (mRNA) (Additional file 1, Table S1). In addition, TCs identify 153 miRNAs out of 154 annotated miRNAs in the miRbase release 12.0 [25]. Interestingly, although all deep-sequencing data were sizefractionated to 18-32 nt, which encompasses the size of mature miRNAs, we found that some precursor miRNAs (pre-miRNAs) have most of their nucleotides covered by TCs (Table 2). This is also observed for other small ncRNAs such as snoRNAs and tRNAs. Most annotated box H/ACA and box C/D snoRNAs, tRNAs and small nuclear RNAs (snRNAs) in FlyBase [26] have overlapping TCs covering 70\% or more of their length, and many are covered by single TCs that cover much of their length (Table 2). Moreover the majority of small non-messenger RNAs (snmRNAs) that have been experimentally validated as stable ncRNA transcripts with as yet uncharacterized functions [27] also have overlapping TCs (Table 2).

\section{TCs differ in their length and tag-depth for different} classes of ncRNAs

Most of the TCs that overlap with well-characterized classes of ncRNAs, such as miRNAs, tRNAs and snoRNAs, have particular length and tag-depth features that are consistent with the features of the corresponding ncRNA classes. TCs overlapping miRNAs show a high peak for the range of 20-30 nt in length (Fig. 2A), while TCs overlapping tRNAs and snoRNAs have bimodal length-distributions (Fig. 2B, C, D). TCs in the range of 60-120 nt overlap almost 70\% of all Drosophila tRNAs. Some of these TCs are longer than the mature corresponding tRNAs, which in Drosophila fall within the range of 60-100 nt. This may well reflect the detection 
Table 2 Coverage of TCs over annotated ncRNAs

\begin{tabular}{lllll}
\hline & annotated & overlapped by TCs (\%) & $\mathbf{2 7 0 \%}$ coverage by TCs $(\%)$ & $\mathbf{2}$ 70\% coverage by single TC (\%) \\
\hline mature miRNA & 154 & $153(99.3)$ & $153(99.3)$ & $153(99.3)$ \\
\hline pre-miRNA & 152 & $151(99.3)$ & $97(63.8)$ & $64(42.1)$ \\
\hline Box H/ACA snoRNAs & 115 & $109(94.8)$ & $102(88.7)$ & $72(62.6)$ \\
\hline Box C/D snoRNAs & 134 & $107(79.9)$ & $104(77.6)$ & $87(64.9)$ \\
\hline tRNAs & 297 & $295(99.3)$ & $284(95.6)$ & $176(59.3)$ \\
\hline snRNAs & 31 & $29(93.5)$ & $27(87.1)$ & $26(83.9)$ \\
\hline snmRNAs & $20^{*}$ & $17(85.0)$ & $8(40.0)$ & $5(25.0)$ \\
\hline
\end{tabular}

*Other 20 snmRNAs that are associated with His gene cluster were excluded due to their repetitive nature.

of uncleaved precursors [28], as these extended TCs had high tag-counts that may have more sensitively detected such precursors.

TCs covering the full length of $53 \%$ of all annotated box C/D snoRNAs and $56 \%$ of annotated box H/ACA snoRNAs (Fig. 2C, D) are within common size ranges of those two classes of snoRNAs (60-100 nt for box C/D snoRNAs and 120-180 nt for box H/ACA snoRNAs in Drosophila). The shorter TCs from tRNAs and snoRNAs that comprise the peaks on the left-hand side of Fig. 2B, $\mathrm{C}$ and 2D may indicate specifically processed short RNAs [21-23] (see Discussion).

Fig. 3 adds another dimension to Fig. 2 by combining the tag-depth feature with the length feature of the TCs within the typical size ranges of corresponding ncRNAs (plotted on top of the unannotated TCs) and clearly discriminates different types of TCs derived from different

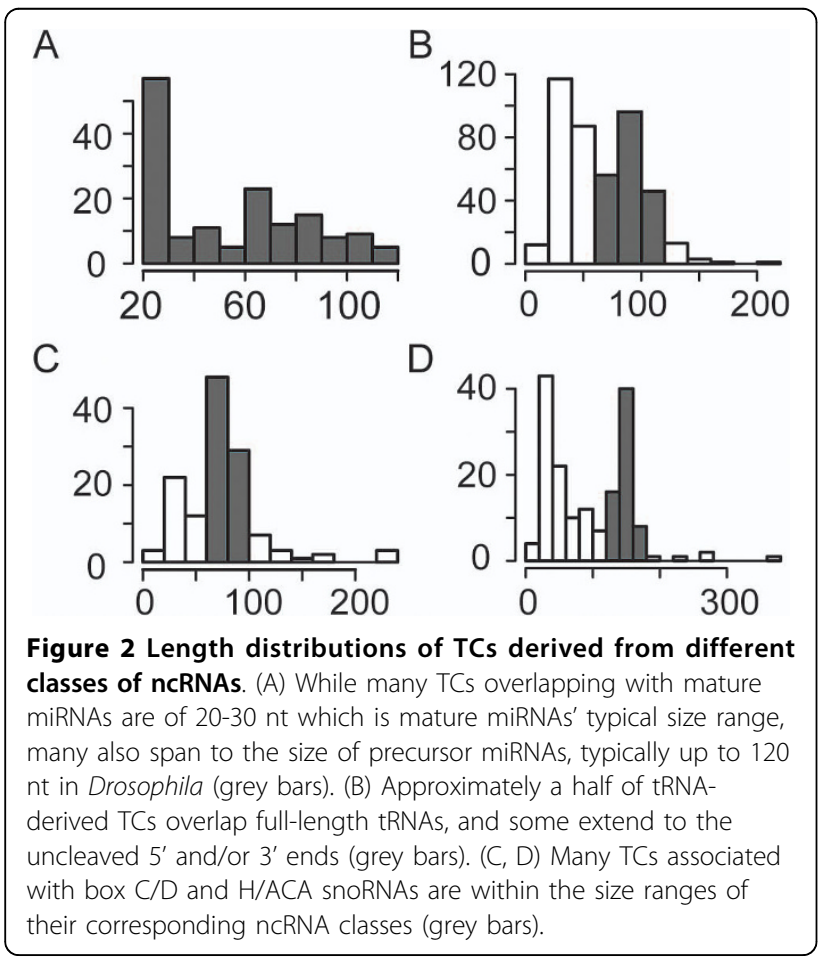

classes of ncRNAs. TCs derived from miRNAs have a wide range of tag-depths (over 4 orders of magnitude) as many miRNAs are differentially expressed in different tissues or developmental stages [2,29]. TCs from the two classes of snoRNAs have similar ranges of tagdepths to each other, while the tag-depths of tRNAderived TCs are generally higher than those of snoRNAderived TCs. There are also many unannotated TCs within the size and tag-depth ranges of snoRNA-derived TCs (Fig. 3), indicating that some may also be unannotated snoRNAs, as well as other TCs that could potentially mark other unannotated ncRNAs. Hence we explored the predictive potential of TCs for the identification of new ncRNA loci.

\section{Filtering of unannotated TCs}

For this analysis we excluded TCs derived from annotated exons, ncRNAs, transposons or other repeats annotated in FlyBase [26] (see Methods). While most of the remaining TCs do not overlap other TCs on the opposite strand, a substantial fraction (21\%) of TCs have overlapping TCs on the other strand. The tag-depths of many of these are not particularly biased to either strand, causing ambiguity in transcription directions, which is characteristic of TCs derived from transposons (Additional file 1, Fig. S1) [9]. In contrast, TCs for known ncRNAs have either no overlapping TCs on the opposite strand or a strong bias in tag-depths towards the sense stand (Additional file 1, Fig. S1). Thus, we further excluded TCs that overlap other TCs on the opposite strands and do not show significantly greater tag-depths than the competing TCs (see Methods), and selected 100,193 TCs for further analysis.

\section{Prediction of seven novel box H/ACA snoRNAs and one SnRNA}

Among the 164 TCs overlapping known box H/ACA snoRNAs (Table 2), 64 are within the size range of 120$180 \mathrm{nt}$, covering full-length or near full-length of box $\mathrm{H} /$ ACA snoRNAs. The tag-depths of those 64 TCs ranged from 15 to 3,308 (Additional file 1, Fig. S2). We also observed that almost all annotated box H/ACA snoRNAs (106 out of 115) are located in annotated introns in the same transcriptional orientation as their host genes, as 


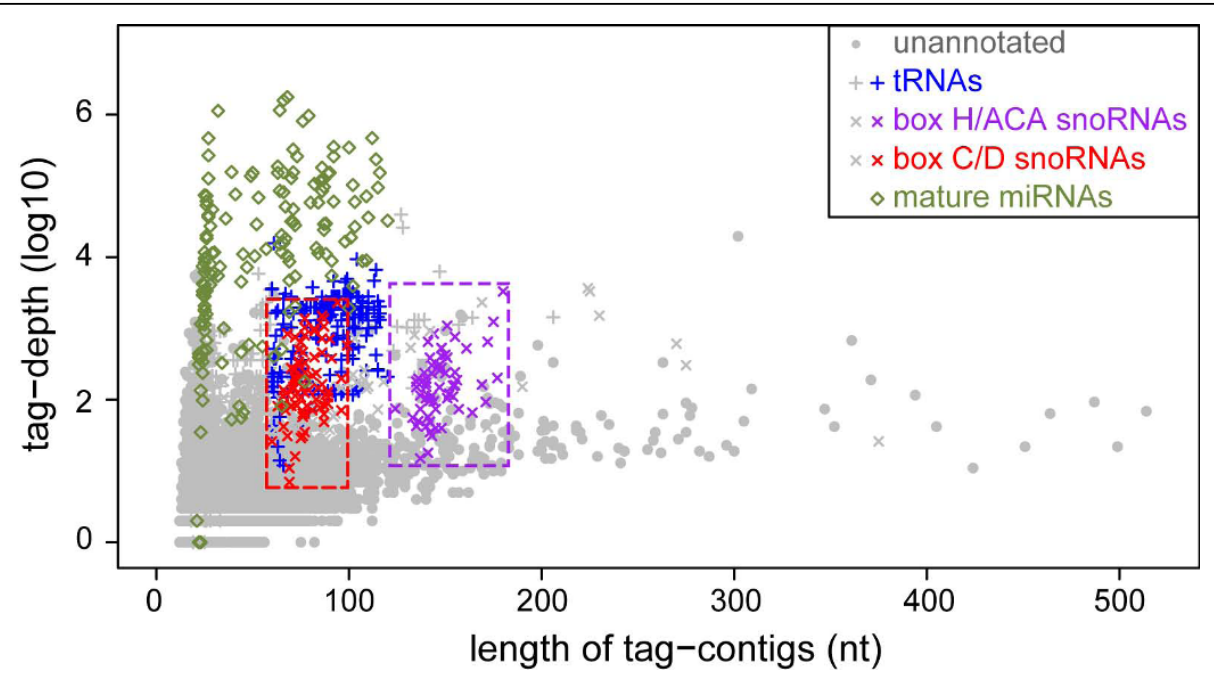

Figure 3 The length and tag-depth distributions of TCs of different categories. TCs overlapping 4 different classes of small ncRNA are plotted on top of all unannotated TCs. TCs comprising grey bars in Fig. 2 are coloured, and they differ in the length and tag-depth according to their corresponding ncRNA classes. While TCs derived from two different classes of snoRNAs have similar range of tag-depths, the TCs of box H/ACA snoRNAs (purple) are much longer than those of box C/D snoRNAs (red). tRNA-derived TCs (blue) overlap the size range with TCs of box C/D snoRNAs but tend to be deeper in tag-depth than box C/D snoRNA-derived TCs. TCs derived from miRNA loci are shown in green and show a wide range of tag-depths. Those spanning precursor miRNAs are scattered within the size ranges of box C/D snoRNAs or tRNAs, but have much greater tag-depths. The dashed boxes around the TCs for box C/D and box H/ACA snoRNAs show the areas within which box C/D and box H/ACA snoRNA candidates were searched for.

expected [30]. Thus, from the 100,193 unannotated TCs, we selected 20 TCs that are (i) intronic (sense), (ii) within size range of 120-180 nt and (iii) with tag-depth of at least 15. Subsequent motif analysis identified 9 of these TCs that have the characteristic box H (ANANNA) and box ACA motifs in the appropriate positions [31] (see Methods). BLAST analysis [32] revealed that one of those 9 TCs, although unannotated in FlyBase, had already been identified as a box H/ACA snoRNA (GenBank AJ809564) (Additional file 1, Table S2), providing a positive control for the analysis. It also showed that one TC at chr3R_1020733_1020883 had a sequence that is almost identical to a snRNA:U4atac:1 in Drosophila simulans (NCBI Reference Sequence XR_050942.1). Considering that most snRNAs in D. simulans were predicted from BLAST analysis with known snRNAs of D. melanogaster against the $D$. simulans genome sequence [33], the TC at chr3R_1020733_1020883 is also a candidate snRNA (Additional file 2). The remaining 7 TCs were classified as novel box H/ACA snoRNA candidates (Additional file 2). Interestingly, the box H/ACA snoRNA candidate TCs tend to have higher tag-depth compared to the $11 \mathrm{TCs}$ excluded due to the absence of motifs. The average and median tag-depths of the 7 candidates TCs are 304 and 105, respectively, while those of excluded TCs are 188 and 44 , respectively.

\section{Prediction of 26 box C/D snoRNAs}

A total of 107 box C/D snoRNAs (out of 134 annotated in FlyBase [26]) are overlapped by $130 \mathrm{TCs}$
(Table 2), of which 78 are within the typical size range of box C/D snoRNAs (60-100 nt). The tag-depth of these TCs ranged from 6 to 2,293 (Additional file 1, Fig. S2). Since box C/D snoRNAs are located either in introns (sense to introns) or in intergenic spacers, we selected those TCs from the 100,193 unannotated TCs that are (i) either intronic or intergenic, (ii) within the size range of 60-100 nt and (iii) with a tag-depth of at least 6. Out of $573 \mathrm{TCs}$ that fulfilled these conditions, we found that 27 have the characteristic box $C$ (RUGAUGA) and box D (CUGA) motifs in the vicinity of their 5' and 3' ends, respectively [31] (see Methods). One of these TCs, at chr3LHet_2398490_2398558, has been previously identified as a snoRNA (GenBank AJ784386) (Additional file 1, Table S2), again providing a positive control for the analysis. The remaining 26 TCs were considered candidate box C/D snoRNAs (Additional file 2). The average tag-depths of these candidate TCs is also much higher than those that do not have recognizable box C/D motifs - 71 and 23 for candidate $\mathrm{TCs}$ and excluded $\mathrm{TCs}$, respectively, although the median tag-depths of those two TC sets are not significantly different from each other - 9 for both. BLAST analysis also showed that one candidate snoCD_05 (chr2L_6917229_6917303) is highly homologous to a box C/D snoRNA, snord53 (GenBank X96652.1), in human and mouse [34] (Additional file 1, Table S2), which subsequently also showed positive in Northern blots (see below). 


\section{TCs for putative ncRNAs}

After these box H/ACA and box C/D snoRNA predictions, 100,157 TCs still remain unannotated. To explore these further, we initially selected 135 highly expressed TCs with a tag-depth $\geq 100$ as Fig. 3 shows that a large portion of TCs mapped to known ncRNAs have tagdepths of 100 or higher. BLAST analysis further excluded 49 TCs that have sequences homologous to annotated transposons. The remaining 86 were classified into three groups based on their length and tag-depth: $8 \mathrm{TCs}$ that are shorter than $40 \mathrm{nt}$ with tag depths of 1000 or more (group 1); 29 TCs that are shorter than $40 \mathrm{nt}$ with tag depths of 100-999 (group 2); and the 49 longer TCs (group 3) (Additional file 1, Fig. S3 and Additional file 3).

The location of group $1 \mathrm{TCs}$ is strongly biased to introns of genes involved in development (Table 3) and with the exception of ncRNA_05, at chrX_3721726_3721755, which is located immediately downstream of a tRNA-Pro locus, all share a consensus 18 nt sequence (18mer motif), GTCCACCCGGGGGCGCCA, which is also by far the most abundant sequence read within these TCs $(31,689$ out of 40,129 reads). Genomic scanning for the $18 \mathrm{mer}$ motif sites found 1 more exact match antisense to the 3' UTR of a gene of uncharacterized function (CG31665). Allowing 1 and 2 mismatches for the genomic scanning identified a further 17 and 31 sites, respectively (excluding chrU, chrUextra and chrM), the majority of which are also intronic (Additional file 1, Table S3). One of the 17 motif sites with 1 mismatch and 9 of the 31 motif sites with 2 mismatches were found at the 3'end of tRNA:N5 (Additional file 1, Table S3) which indicates that this motif may have arisen from tRNA:N5. However, none of the sequence tags contributing to the 7 TCs is mapped to the tRNA:N5, suggesting that these sequence tags are produced independently of tRNAs. The 7 Group 1 TCs sharing the 18mer motif show high expression levels in S2 and Kc cell lines, while the tRNA-associated TC has a significantly high number of sequence reads from the midembryonic stages (Additional file 1, Fig. S4).

The 29 TCs in Group2 are evenly distributed in introns (13 TCs) and intergenic spacers (16 TCs), and intronic TCs and intergenic TCs do not differ in tagdepth distributions. Two of these TCs, ncRNA_12 and ncRNA_37, were detected by genomic scanning for the 18 mer motif derived from the Group 1 TCs when 1 mismatch was allowed. Those two TCs along with ncRNA_15 are mainly composed of sequence reads specifically obtained from S2 cells [35] (Additional file 1, Fig. S5). Two other TCs, ncRNA_20 and ncRNA_28, are composed of sequence reads heavily biased to adult body, larvae, and pupae (Additional file 1, Fig. S5).

In Group3, 3 of the 49 TCs were found to be derived from annotated ncRNAs by BLAST analysis (Additional file 1, Table S4). Those at chr2R_7292203_7292273 (nRNA_48) and chr2R_7292691_7292839 (ncRNA_49) have recently been annotated as tRNAs-Thr in the Genomic tRNAdb [36] and that at chr3R_2645849_2646151 (ncRNA_60) defines 7SL RNA precisely [37], further demonstrating that this approach is able to detect various types of small ncRNAs (Additional file 1, Table S4). We also found that a cluster of $17150 \mathrm{nt}$-long TCs located within a $4.6 \mathrm{~kb}$ region, chrX:4,815,890-4,820,490, is part of an endogenous siRNA cluster identified by Czech et al. [13] (Additional file 1, Table S4). Each of the TCs in this cluster is an exact copy of the others and forms a hairpin structure which is the precursor of siRNAs. The remaining 29 TCs are not particularly enriched in introns, but intronic TCs tend to have higher tag-depths than intergenic TCs (Additional file 1, Table S5).

\section{Experimental validation of putative ncRNAs}

For experimental validation of snRNA and snoRNA predictions, we selected the snRNA candidate along with top 4 and 5 box H/ACA and box C/D snoRNA candidates, respectively, based on the tag-depth. The snRNA candidate, all 4 box H/ACA snoRNA candidates and 3 of the box C/D snoRNA candidates tested positive by Northern blot (Table 4) (Fig. 4A, B), with clear bands of the approximately expected sizes based on the length of TCs. The 2 candidates that were not experimentally confirmed have relatively low tag-depths (27 each) compared to the others (at least 42), suggesting sensitivity

Table 3 TCs in Group1, unannotated but highly expressed TCs.

\begin{tabular}{|c|c|c|c|c|c|}
\hline & locus & tag-depth & strand & TC size $(\mathrm{nt})$ & Gene \\
\hline ncRNA_01 & chr2R_4733783_4733804 & 5027 & + & 21 & sns \\
\hline ncRNA_02 & chr2R_9632216_9632238 & 6051 & - & 22 & fas \\
\hline ncRNA_03 & chr2R_13693470_13693490 & 5460 & - & 20 & grh \\
\hline ncRNA_04 & chr2R_19535102_19535122 & 5460 & - & 20 & retn \\
\hline ncRNA_06 & chrX_11524384_11524406 & 5685 & + & 22 & Ptp10D \\
\hline ncRNA_07 & chrX_12399632_12399653 & 5697 & - & 21 & CG2556 \\
\hline ncRNA_08 & chrX_19880356_19880381 & 6749 & + & 25 & $\mathrm{~N} / \mathrm{A}$ \\
\hline nCRNA_05* & chrX_3721726_3721755* & 4733 & + & 29 & ec \\
\hline
\end{tabular}

*Located downstream of a tRNA. 
problems and/or that the snoRNAs are not expressed in the cell line or embryonic stages tested (see Methods). In fact, while all confirmed snoRNA candidates have large number of sequence reads from late embryo (12$18 \mathrm{~h}$ ) and S2 cells (Additional file 1, Fig. S6A, B) on which the experimental validations were performed (see Methods), the two unconfirmed box C/D snoRNA candidates have the highest number of sequence reads from early embryo (Additional file 1, Fig. S6B).

We also carried out Northern blots using the $18 \mathrm{mer}$ motif that dominates the tag spectrum in 7 out of 8 Group1 TCs, and observed a strongly hybridizing band of approximate size $21 \mathrm{nt}$ along with weaker $18 \mathrm{nt}$ and 26 nt bands in S2 cells with a different distribution in late-embryo (Fig. 4C). We also observed larger $42 \mathrm{nt}, 48$ nt and 79 nt bands, the latter of which (and perhaps others) may well be the result of cross-hybridization to highly abundant RNA molecules such as tRNAs, given the extremely high GC content of the 18 mer motif $(83.3 \%)$ and the high similarity to tRNA:N5 (Additional file 1, Table S3). In any case, the bands between 18 and $26 \mathrm{nt}$ clearly suggest that the $18 \mathrm{mer}$ motif is expressed as small RNAs, and is consistent with the incidence and size of the tags covering this motif.

A subset of group 3 TCs was also tested by Northern blot. As the majority of TCs for known ncRNAs overlap phastCons elements [38] (Additional file 1, Table S6) (see Methods), we selected 6 intronic and 4 intergenic TCs that mapped to phastCons elements, 5 and 1 of which, respectively, showed positive in Northern blots (Table 4) (Fig. 4D). Two exhibited clear bands of the approximately expected sizes based on the TC lengths, whereas the other four exhibited bands that are either shorter or longer than the lengths of their corresponding TCs (Table 4). For the three TCs with shorter sized bands, ncRNA_47 (chr2R_4612441_4612565), ncRNA_54 (chr3L_1488738_1488831) and ncRNA_39 (chr2L_8485729_8485927), the number of overlaying reads is very low in a few parts of each, suggesting that those TCs may represent unprocessed precursors (Additional file 1, Fig. S7). On the other hand, an intronic TC ncRNA_64 (chr3R_24973624_24973672) showed a very weak band of approximately 70 bp (data not shown), while its expected length was $48 \mathrm{nt}$ (Table 4).

Table 4 Experimental validation results for selected novel ncRNA candidates.

\begin{tabular}{|c|c|c|c|c|c|}
\hline & Type & tag-depth & TC size (nt) & Estimated size from Northern blot (nt) & Gene \\
\hline snoHACA_01 & box H/ACA snoRNA & 105 & 145 & $\sim 150$ & SC35 \\
\hline snoHACA_04 & box H/ACA snoRNA & 234 & 143 & $\sim 130$ & Dek \\
\hline snoHACA_05 & box H/ACA snoRNA & 106 & 137 & $\sim 170$ & hts \\
\hline snoHACA_06 & box H/ACA snoRNA & 1555 & 158 & $\sim 130$ & CG31191 \\
\hline snRNA_01 & snRNA:U4atac & 282 & 150 & $\sim 150$ & cno \\
\hline snoCD_01 & box C/D snoRNA & 27 & 74 & N/A & $\mathrm{N} / \mathrm{A}$ \\
\hline snoCD_02 & box C/D snoRNA & 42 & 81 & $\sim 85$ & kis \\
\hline snoCD_05 & box C/D snoRNA & 566 & 74 & $\sim 70$ & $x 16 /$ nop \\
\hline snoCD_09 & box C/D snoRNA & 27 & 80 & N/A & N/A \\
\hline snoCD_24 & box C/D snoRNA & 993 & 69 & $\sim 59$ & N/A \\
\hline ncRNA_01* & Group1 & 5027 & $20 \sim 25^{\dagger}$ & $18 / 21 / 26$ & $\neq$ \\
\hline ncRNA_38 & Group3 (intron) & 1418 & 159 & $\sim 150$ & $v(2) k 05816$ \\
\hline ncRNA_39 & Group3 (intron) & 582 & 198 & $\begin{array}{l}\sim 90 / \sim 190 \\
\end{array}$ & $D$ \\
\hline ncRNA_47 & Group3 (intron) & 418 & 124 & $\sim 70$ & gcl \\
\hline ncRNA_54 & Group3 (intron) & 153 & 93 & $\sim 49 / \sim 55$ & Stet \\
\hline ncRNA_64 & Group3 (intron) & 223 & 48 & $\sim 70$ & CG11882 \\
\hline ncRNA_85 & Group3 (intron) & 225 & 72 & $\mathrm{~N} / \mathrm{A}$ & CG1718 \\
\hline ncRNA_50 & Group3 (intergenic) & 106 & 172 & $\sim 180$ & N/A \\
\hline ncRNA_55 & Group3 (intergenic) & 180 & 79 & $\mathrm{~N} / \mathrm{A}$ & N/A \\
\hline ncRNA_62 & Group3 (intergenic) & 123 & 56 & N/A & N/A \\
\hline ncRNA_83 & Group3 (intergenic) & 116 & 394 & N/A & $\mathrm{N} / \mathrm{A}$ \\
\hline
\end{tabular}

*One of the 7 TCs in Group1 that shares an 18 nt long sequence. ${ }^{\dagger}$ ncRNA_05 which is immediately downstream of a tRNA was excluded. ${ }^{*}$ Introns of 6 genes shown in Table 3. 


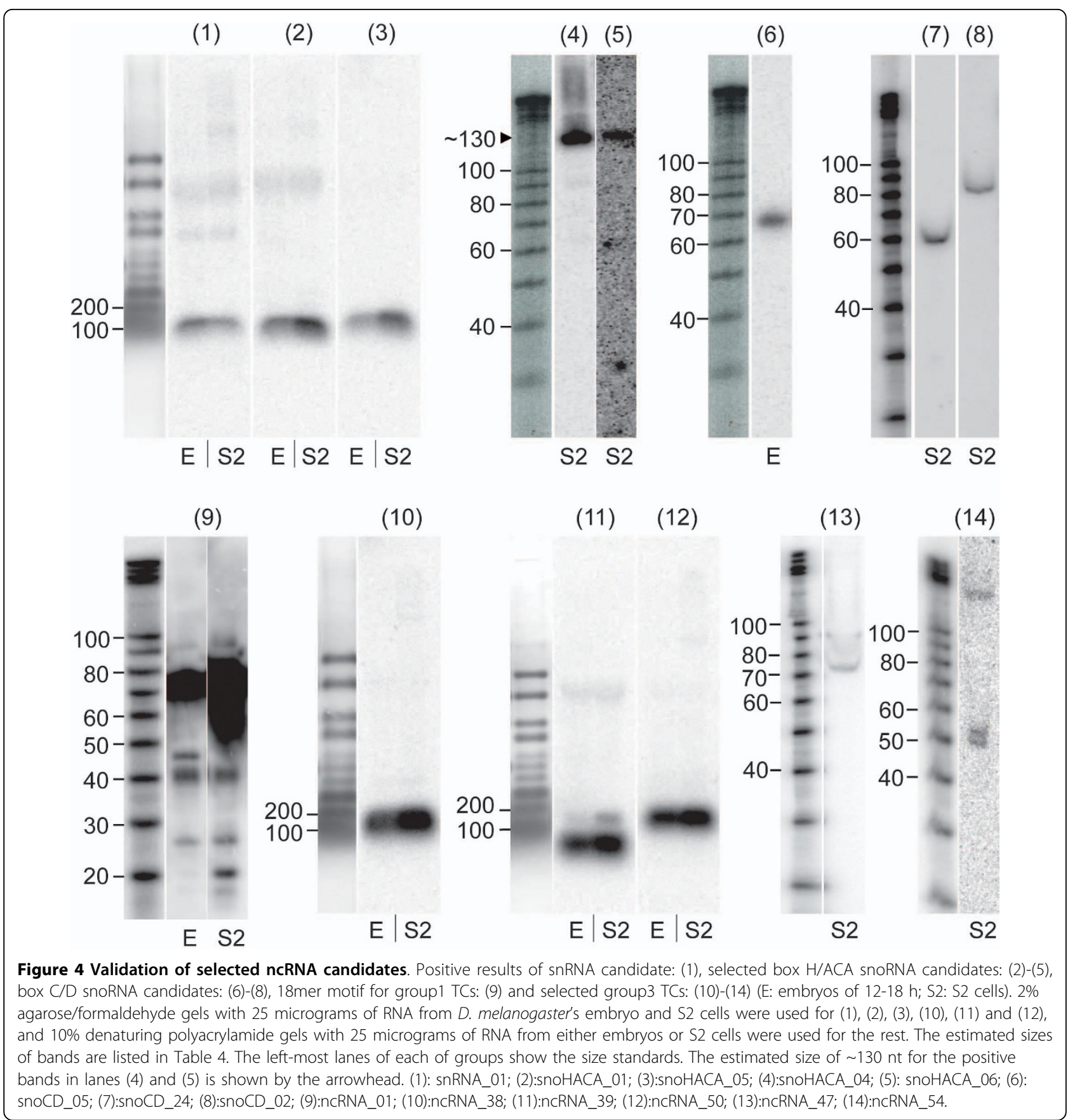

Considering that this $\mathrm{TC}$ is located within a $70 \mathrm{nt}$ long intron of CG11882, it may be that the actual transcript detected by Northern covers the entire intron (Additional file 1, Fig. S8). The numbers of sequence reads for TCs with weak signals, ncRNA_54 (chr3L_1488738_1488831) (Fig. 4D) and ncRNA_64 (chr3R_24973624_24973672) (data not shown), were lower than those of the other confirmed TCs, and the most reads for those two TCs were obtained from midembryonic stages (Additional file 1, Fig. S6C). This could be the reason for the weak signals from Northern blot as is for the unconfirmed box C/D snoRNA candidates. The 4 unconfirmed TCs also have small number of sequence reads from late embryos and S2 cells, while most of their sequence reads are from early and midembryonic stages (Additional file 1, Fig. S6C).

Probe sequences for the Northern blotting analysis are provided in Additional file 4, and the sequences of validated ncRNA candidates are provided in FASTA format in Additional file 5. 


\section{Discussion}

In this study, we utilized a strategy of analyzing millions of short reads from next generation sequencing experiments for the prediction of novel ncRNAs of both known and unknown classes. Although the deepsequencing analyses used in this study focus on identifying shorter ncRNAs such as miRNAs, siRNAs and piRNAs by limiting the lengths of the RNA samples to the sizes of such small ncRNAs, assemblages of contiguously overlapping tags also overlap with longer ncRNAs such as snoRNAs, snRNAs and tRNAs.

TCs derived from two different classes of snoRNAs showed distinct features in their length and tag-depth distributions, and the use of these characteristic features along with the their signature motifs predicted novel snoRNAs. Proof-of-principle of this approach is provided by the successful recall of two previously known but not FlyBase-annotated snoRNAs as well as the de novo identification of three known ncRNAs (two tRNAs and 7SL RNA) and an endogenous-siRNA cluster. We also found that the majority of experimentally detected snmRNAs [27] (excluding those that are related to His clusters) are overlapped by TCs, another demonstration of the validity of the approach. In fact, one TC (chr3R_3300274_3300719) overlapping snmRNA:331 corresponds to the 7SK RNA recently identified in Drosophila [39], the boundaries of which fit better to the 5' and 3' ends of the TC than those of snmRNA:331.

Characteristic features of snoRNAs were extracted from TCs that cover the full-length of annotated snoRNAs. However, there are also many short TCs partially overlapping with annotated snoRNAs, with strong positional preference in both 5'/3' ends of snoRNAs, which is consistent with the positional preferences of snoRNAderived small RNAs (sdRNAs) [22]. These positional preferences were also observed and used for novel snoRNA predictions in the Arabidopsis genome [24]. We also found that these short TCs within snoRNAs were closely juxtaposed. Thus, more accumulation of deep-sequencing data would be expected to connect these TCs and identify more novel snoRNAs. We also examined the potential of making a simple merge of closely located TCs but this approach was compromised by also merging adjacent snoRNAs. Chen et al. [24] bypassed this problem in their snoRNA predictions by first anchoring the 3' ends of the novel snoRNA transcripts and then looking for their 5' ends. However, this method cannot be easily generalized for ncRNAs of uncharacterized classes. Alternatively, carefully designed computational approaches using the distribution of short RNA tags across annotated snoRNAs may also increase the number of novel snoRNAs predictions. Our candidates were tested by the snoRNA prediction software SnoReport, which also refuses to use the modification target information of snoRNAs [40], but it identified (using the default options) only 3 box H/ACA and 5 box C/D snoRNAs from our 7 and 26 snoRNA candidates, respectively. However, when we tested the performance of SnoReport on the 115 box H/ACA and 134 box C/D snoRNAs that are annotated in the Drosophila melanogaster genome, only 59 box H/ACA and 51 box C/D snoRNAs were successfully recalled.

Unlike the prediction of box C/D snoRNAs and putative ncRNAs of uncharacterized classes, the box H/ACA snoRNA prediction incorporated another filter that excluded non-intronic TCs. This was based on the fact that $92 \%$ of the known box H/ACA snoRNAs reside in introns, and reduced the number of predictions from 18 (based on tag-contig size, tag depth and presence of the $\mathrm{H} / \mathrm{ACA}$ motif) to 7 . It is uncertain how many of the 10 discarded TCs (excluding one snRNA candidate) may be genuine box H/ACA snoRNAs, but the high validation rate of the intronic subset (4 out of 4 tested) indicates that the incorporation of the location filter improved the specificity of the prediction.

The length and tag-depth distributions of unannotated TCs are similar to those of exon-derived TCs (Additional file 1, Fig. S9A, B), which may indicate that some unannotated TCs might be assemblages of degradation products of unknown exons. However, it is equally possible that they may also represent degraded or processed fragments of bona fide ncRNAs that can also be reassembled, as is evidently the case for snoRNAs. Moreover, the large amount of unannotated TCs located in introns and intergenic regions (Additional file 1, Fig. S9C, D) indicates that there are many more unknown transcripts yet to be investigated. Considering that we used a conservative threshold of tag-depths $(\geq 100)$ for uncharacterised ncRNA candidates as the vast majority of exon-derived TCs (99.9\%) have tag-depths less than 100 , the novel ncRNA candidates shown in this study are just the tip of the iceberg. We tested 10 of the 29 putative ncRNA candidates in group 3 , focusing on those that were most highly conserved, 6 of which returned positive signals in a restricted range of cells (see below). However, considering that some ncRNAs evolve at high rate [41], the untested 19 ncRNA candidates in group 3 could equally likely be novel ncRNAs. Indeed, among the total of 100,193 unannotated TCs, only 26,395 overlap phastCons elements, and, surprisingly, there is no apparent difference in the distributions of lengths and tag-depths between TCs that overlap conserved sequences and those do not (Additional file 1, Fig. S9E, F). This suggests that while conservation may be used as a positive guide to likely ncRNAs, the relative lack of conservation is not necessarily an index of lack of relevance of others. 
In the experimental validation, 15 out of 21 selected candidate TCs were positive -8 out of 10 selected snRNA and snoRNA candidates $(80 \%)$ and 7 of 11 (64\%) putative unclassified transcripts of either group 1 or group 3. This is a high rate of validation, given that the likelihood of detecting a signal in Northern blots is dependent on the expression level of the candidate in the tissue concerned. In fact, the confirmed candidates have generally greater tag-depths than the unconfirmed (Table 4), and they are also more contributed by tags obtained from late embryos or S2 cells (Fig. 5) that were the source material for Northern blots. In contrast, among the confirmed candidates, the TCs with weaker signals have a lower number of sequence reads from late embryonic stages than other confirmed candidates (Fig. 5).

In addition, there remain a large set of $\sim 27,000 \mathrm{TCs}$ that overlap TCs on complementary strands, which is characteristic of TCs mapping to transposons. They are also closely located to each other $(\leq 100 \mathrm{bp})$, similar to TCs covering known transposon-derived sequences, and different to the $\sim 100,000$ TCs which were used for this study. We also observed that a large portion (37\%) of these $27,000 \mathrm{TCs}$ is found within reported siRNA/ piRNA clusters $[8,9,13,14,20,42]$. Although some of siRNA or piRNA clusters are not associated with transposons [13], these preliminary observations indicate that some of these complementary TCs may be derived from unidentified transposons. In fact, about five thousand TCs in this set either slightly overlap with or are located close to $(\leq 100 \mathrm{bp})$ existing transposons have sequences homologous to transposons, suggesting they could be unannotated parts of existing transposons generating siRNAs or piRNAs.

\section{Conclusions}

Several studies investigating the population of small RNAs have yielded millions of sequence reads. In this study, we combined all publicly available sequence data from Drosophila melanogaster short RNA into hundreds of thousands tag-contigs and associated subsets of them with known ncRNAs such as snoRNAs and tRNAs. The characteristic features of TCs overlapping with known ncRNAs were used to predict 7 and 26 box H/ACA and box C/D snoRNA candidates, respectively, in addition to one snRNA and many novel unclassified ncRNA candidates, a substantial fraction of which were experimentally validated. We conclude that deep sequencing from short reads may be used to identify new members of known and novel classes of ncRNAs, including those that are significantly longer than the reads themselves.

\section{Methods}

\section{Genome sequence and annotation}

We used the D. melanogaster genome sequence assembly Release 3 (April, 2006) from the Berkeley Drosophila

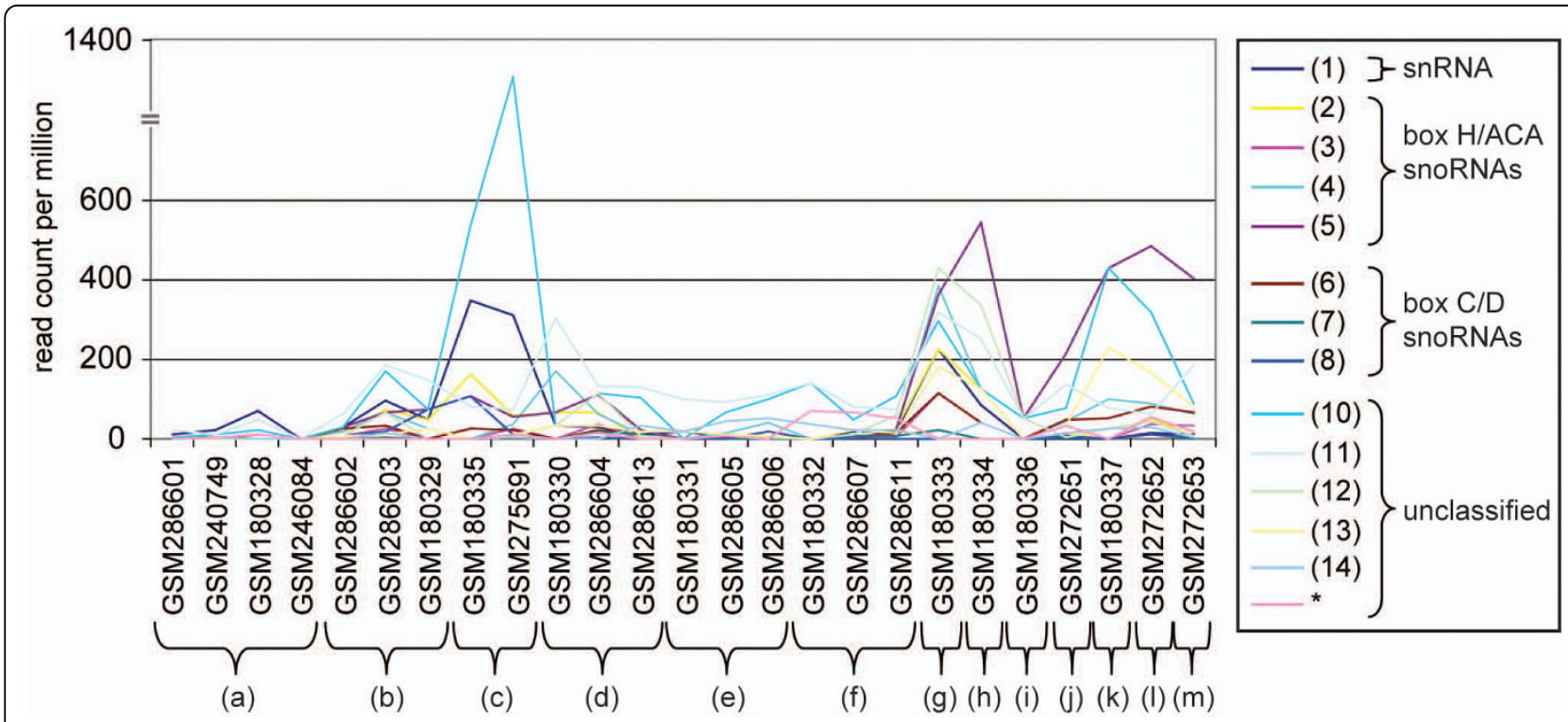

Figure 5 Number of sequences per million from each experiment for confirmed TCs. (a) heads, (b) adult body, (c) imaginal discs, (d) very early embryo (0-1 h), (e) early embryo (2-6 h), (f) mid embryo (6-10 h), (g) late embryo (12-24 h), (h) larvae: 1st instar and 3rd instars, (i) pupae: 0-1 day, 0-2 day, 2-4 day, (j) S2 and KC cells, (k) tissue culture cells (S2 only), (I) S2 cells, (m) KC cells. Numbers for TCs shown on right panel are correspondent to those in Fig. 4 (*: not shown in Fig. 4). Overall they show similar expression profiles in different tissues of developmental stages. Expression profiles of Group1 TCs including ncRNA_01 are shown in Additional file 1, Fig. S4. Expression profiles of all tested TCs are shown in Additional file 1, Fig. S5. 
Genome Project. Annotation of exons, introns, UTRs, and ncRNAs are from FlyBase 5.12 [26]. MicroRNA annotation was obtained from miRbase release 12.0 [25]. Repeats were annotated using RepeatMasker [43] in FlyBase 5.12.

\section{Mapping of sequence tags}

We obtained all public available deep-sequencing datasets from Gene Expression Omnibus database at National Center for Biotechnology Information http:// www.ncbi.nlm.nih.gov/geo in SOFT format (Table 1). These sequences were subsequently mapped to the genome of D. melanogaster using Vmatch http://www. vmatch.de and a bioinformatics toolkit - Biopieces http://www.biopieces.org, to obtain all full length exact hits. Hits on chrM, chrU and chrUextra were discarded. Each tag in the dataset is comprised of a number of reads, i.e., the number of times the tag was sequenced. For tags mapping to unique locations in the genome, it is obvious that the mapped locus was cloned and sequenced as many times as the number of reads of the given tag. For tags mapping to multiple loci, the number of reads of the given tag was distributed evenly to each mapped locus, and transcripts from each locus were assumed to have been cloned and sequenced as many times as the number of reads of the tag divided by the number of mapped loci [8]. Sequence tags that had a greater number of mapped loci than the number of reads were discarded.

\section{Tag contigs}

A tag-contig (TC) is defined as a genomic region that has mapped sequence tags with the same strand orientation contiguously overlapping with each other by at least $1 \mathrm{nt}$ (Fig. 1). Each base within a TC is overlayed by the number of reads that include the base (adjusted number of reads for multi-mapping tags), and the maximum accumulation of read numbers within a given $\mathrm{TC}$ is defined as the tag-depth for the TC (Fig. 1).

\section{Classification of TCs}

TCs overlapping at least $20 \%$ of the length with exons, introns, repeats and annotated ncRNAs were classified as TCs derived from each of the annotations. TCs have less than $20 \%$ overlap with exons, genic regions and repeats were regarded as non-exonic (or intronic), intergenic and non-repeat TCs, respectively. Intersection of TCs with other annotation was performed through local mirror of University of California, Santa Cruz Genome Browser [44].

\section{Selection of TCs for analysis}

Among the total of 521,302 TCs, 126,962 are outside of annotated exons, ncRNAs, transposons and other repeats annotated in FlyBase [26]. Of these, 27,151 TCs overlap with other TCs mapped to the complementary strand. Most TCs sense to known ncRNAs have at least 10 times greater tag-depth than those that are antisense to known ncRNAs (Additional file 1, Fig. S1). Based on this observation of fold-differences, we selected 382 from the $\sim 27,000 \mathrm{TCs}$, that have at least 10 times greater tag-depth than their overlapping TCs on the opposite strand.

\section{Scatter plotting of TCs}

The scatter-plots of lengths (nt) against tag-depth ( $\log 10)$ of TCs were generated by R http://www.R-project.org and in-house software along with the bioinformatics toolkit, Biopieces http://www.biopieces.org.

\section{Conservation of sequence reads}

A sequence tag overlapping with phastCons elements [38] by at least $15 \mathrm{bp}$ is considered as a conserved sequence tag. Each sequence tag represents a number of sequence reads, thus sequence reads comprising the conserved tags are also regarded as conserved reads.

\section{Motifs in snoRNA candidate TCs}

For each of the unannotated TCs within the ranges of length and tag-depth of box C/D snoRNA-derived TCs, box C motif (RUGAUGA) and box D motif (CUGA) were searched within $+/-10$ bp from the 5 ' end and within $+/-10$ bp from the 3 ' end, respectively [40]. One mismatch was allowed for both box $C$ and D motifs. For the box H/ACA snoRNA predictions, $20 \mathrm{bp}$ of flanking sequences of the midpoint of a TC were searched for the box $\mathrm{H}$ motif (ANANNA), and $20 \mathrm{bp}$ of flanking sequence of 3' end of a TC were examined for the box ACA motif [40].

\section{Gene Ontology analysis}

The Gene Ontology term enrichment analyses in this study were performed using GO-TermFinder [45] through the AmiGO web site http://amigo.geneontology. org/cgi-bin/amigo/term_enrichment.

\section{Northern blots}

Total RNA was extracted from Drosophila's late embryos (12-18 h) and S2 cells using TRIZOL reagent (Invitrogen). Fifteen micrograms of total RNA was separated on $1 \%$ denaturing agarose gels, and 25 micrograms of total RNA was on $2 \%$ agarose/formaldehyde gels and $10 \%$ denaturing polyacrylamide gels. RNA separated using denaturing agarose was then transferred to Hybond-N+ membranes (GE Healthcare) using downward capillary transfer, then UV-crosslinked and baked at $80^{\circ} \mathrm{C}$ for 1 hour. RNA separated by denaturing polyacrylamide gels was transferred to Hybond-Nx membranes (GE Healthcare) by use of a semidry transfer cell apparatus, and cross-linked using the EDC method as outlined in [46]. Antisense oligonucleotides complementary to predicted ncRNA candidates were used as probes. Northern blotting was carried out as described by Nelson Lau from Bartel Laboratory, http://web.wi. mit.edu/bartel/pub/protocols/miRNA_Nrthrns_Protocol. pdf. In brief, the pre-hybridization/hybridization buffer contained 5× SSC, $20 \mathrm{mM} \mathrm{Na2HPO} 4 \mathrm{pH}$ 7.2, 7\% SDS, 
and $2 \times$ Denhardt's solution. Blots were pre-hybridized for at least 2 hours at $50^{\circ} \mathrm{C}$, then probes which had been end-labeled with $\gamma$-32P ATP by use of T4 polynucleotide kinase (New England Biolabs), or end-labeled with $\alpha$ 32P dCTP by use of terminal transferase (New England Biolabs), were added to the hybridization chamber and incubated with the blots overnight at $50^{\circ} \mathrm{C}$. After three washes with non-stringent wash buffer containing $3 \times$ SSC, $25 \mathrm{mM} \mathrm{NaH2PO} 4 \mathrm{pH}$ 7.5, and 5\% SDS, blots were given a final wash with $1 \times$ SSC and 1\% SDS. The membrane was then exposed to a phosphoimager overnight and scanned.

\section{Secondary structure analysis}

The secondary structures of 7 box H/ACA snoRNA candidates (Additional file 6) were predicted by RNAfold [47]. In the case of snoHACA_07 (at chrX_915376_915513), the 5' and 3' ends were extended by $10 \mathrm{bp}$ to include the box ACA motif.

Additional file 1: Supplementary figures and tables. This file contains supplementary figures S1-S9 and tables S1-S6 in PDF format. Fig. S1: The distributions of relative tag-depths of TCS against oppositely stranded overlapping TCs; Fig. S2: Tag-depth distributions of TCs covering fulllength ncRNAs; Fig. S3: Length and tag-depth distribution of highly expressed unannotated TCs; Fig. S4: Number of sequence reads per million per different samples for Group1 TCs; Fig. S5: Number of sequence reads per million per different samples for Group2 TCs; Fig. S6: Number of sequence reads per million per sample for each of tested snoRNA and unclassified ncRNA candidates; Fig. S7: The accumulation of sequence reads per each base across the three TCs; Fig. S8: Screenshot of UCSC genome browser for ncRNA_64, an intronic TC at chr3R_24973624_24973672; Fig. S9: Length and tag-depth distributions of TCs of different categories; Table S1: Coverage of TCs over transposons and exons; Table S2: Predictions of snoRNA candidates; Table S3: 18mer motif sites derived from group1 TCs with 0, 1 and 2 mismatches; Table S4: Group3 TCs mapped to existing ncRNAs; Table S5: 29 unannotated TCs in group3; Table S6: Conservation of sequence tags comprising ncRNA-derived TCs.

Click here for file

[http://www.biomedcentral.com/content/supplementary/1471-2164-1177-S1.PDF ]

Additional file 2: List of all snRNA, box C/D and box H/ACA snoRNA candidates. This file contains genomic loci, strand, tag-depths and predicted lengths of 7 box H/ACA snoRNA candidates, 26 box C/D snoRNA candidates and 1 snRNA candidate.

Click here for file

[http://www.biomedcentral.com/content/supplementary/1471-2164-1177-S2.XLS]

Additional file 3: List of all group1, group2 and group3 TCs. This file contains genomic loci, strand, tag-depths and predicted lengths of all putative ncRNAs of uncharacterized classes: 8 group1 ncRNAs, 29 group2 ncRNAs and 49 group3 ncRNAs.

Click here for file

[http://www.biomedcentral.com/content/supplementary/1471-2164-1177-S3.XLS ]

Additional file 4: List of probes for Northern blot. This file contains probe sequences for experimental validation of 4 box H/ACA snoRNA candidates, 5 box C/D snoRNA candidates, 1 snRNA candidate and 10 putative ncRNA of uncharacterized class along with their other information such as genomic loci, predicted lengths and tag-depths. Click here for file

[http://www.biomedcentral.com/content/supplementary/1471-2164-1177-S4.XLS ]
Additional file 5: Sequences of experimentally validated novel snoRNAs and unclassified ncRNA candidates. This file contains DNA sequences of all experimentally validated ncRNAs: 4 box H/ACA snoRNAs, 3 box C/D snoRNAs, 1 snRNA and 13 ncRNAs of uncharacterized class. Click here for file

[http://www.biomedcentral.com/content/supplementary/1471-2164-1177-S5.SEQ]

Additional file 6: Sequence conservation and secondary structure predictions of snoRNA candidates. This file contains UCSC genome browser screenshots of 7 box H/ACA snoRNA candidates and 26 box C/ D snoRNA candidates showing sequence conservation and tag distributions over each candidate. The positions of box H, ACA, C and D motifs are indicated by the red boxes. Predicted secondary structures of box H/ACA snoRNA candidates are provided below each of the screenshots.

Click here for file

[http://www.biomedcentral.com/content/supplementary/1471-2164-1177-S6.PDF ]

\section{Acknowledgements}

We thank Kelin Ru for assistance with the Northern blots, and Ryan Taft and Harald Oey for helpful comments and discussions. This work was supported by the Australian Research Council (grant FF0561986), the University of Queensland, and the Queensland State Government.

\section{Author details}

'Institute for Molecular Bioscience, University of Queensland, St Lucia QLD 4072, Australia. ${ }^{2}$ Department of Molecular and Cellular Biology, Institute of Chemical Biology and Fundamental Medicine, 630090 Novosibirsk, Russia.

\section{Authors' contributions}

CJ contributed to the study design, carried out the data analysis and drafted the manuscript. MAH contributed to the study design and carried out the data collection and preparation. IVM contributed to the study design and helped draft the manuscript. DJK carried out the experimental validations. JSM supervised the study and helped write the manuscript. All authors read and approved the final manuscript.

\section{Authors' Information}

IVM current address: Department of Molecular and Cellular Biology, Institute of Chemical Biology and Fundamental Medicine, 630090 Novosibirsk, Russia.

Received: 19 June 2009

Accepted: 1 February 2010 Published: 1 February 2010

\section{References}

1. Reinhart BJ, Slack FJ, Basson M, Pasquinelli AE, Bettinger JC, Rougvie AE, Horvitz HR, Ruvkun G: The 21-nucleotide let-7 RNA regulates developmental timing in Caenorhabditis elegans. Nature 2000, 403:901-906.

2. Bartel DP: MicroRNAs: Genomics, biogenesis, mechanism, and function. Cell 2004, 116:281-297.

3. Kim VN: MicroRNA biogenesis: Coordinated cropping and dicing. Nat Rev Mol Cell Biol 2005, 6:376-385.

4. Kloosterman WP, Steiner FA, Berezikov E, de Bruijn E, Belt van de J, Verheul M, Cuppen E, Plasterk RHA: Cloning and expression of new microRNAs from zebrafish. Nucl Acids Res 2006, 34:2558-2569.

5. Berezikov E, van Tetering $G$, Verheul M, Belt van de J, van Laake L, Vos J, Verloop R, Wetering van de M, Guryev V, Takada S, et al: Many novel mammalian microRNA candidates identified by extensive cloning and RAKE analysis. Genome Res 2006, 16:1289-1298.

6. Fahlgren N, Howell MD, Kasschau KD, Chapman EJ, Sullivan CM, Cumbie JS, Givan SA, Law TF, Grant SR, Dangl JL, et al: High-throughput sequencing of Arabidopsis microRNAs: Evidence for frequent birth and death of miRNA genes. PLOS ONE 2007, 2:e219.

7. Aravin AA, Hannon GJ, Brennecke J: The Piwi-piRNA pathway provides an adaptive defense in the transposon arms race. Science 2007, 318:761-764. 
8. Brennecke J, Aravin AA, Stark A, Dus M, Kellis M, Sachidanandam R, Hannon GJ: Discrete small RNA-generating loci as master regulators of transposon activity in Drosophila. Cell 2007, 128:1089-1103.

9. Ghildiyal M, Seitz H, Horwich MD, Li C, Du T, Lee S, Xu J, Kittler ELW, Zapp ML, Weng Z, et al: Endogenous siRNAs derived from transposons and mRNAs in Drosophila somatic cells. Science 2008, 320:1077-1081.

10. Zamore PD, Tuschl T, Sharp PA, Bartel DP: RNAi: Double-stranded RNA directs the ATP-dependent cleavage of mRNA at 21 to 23 nucleotide intervals. Cell 2000, 101:25-33.

11. Aliyari R, Wu Q, Li H-W, Wang X-H, Li F, Green LD, Han CS, Li W-X, Ding SW: Mechanism of induction and suppression of antiviral immunity directed by virus-derived small RNAs in Drosophila. Cell Host Microbe 2008, 4:387-397.

12. Chung W-J, Okamura K, Martin R, Lai EC: Endogenous RNA interference provides a somatic defense against Drosophila transposons. Current Biol 2008, 18:795-802.

13. Czech B, Malone CD, Zhou R, Stark A, Schlingeheyde C, Dus M, Perrimon N, Kellis $M$, Wohlschlegel JA, Sachidanandam $R$, et al: An endogenous small interfering RNA pathway in Drosophila. Nature 2008, 453:798-802.

14. Kawamura Y, Saito K, Kin T, Ono Y, Asai K, Sunohara T, Okada TN, Siomi MC, Siomi H: Drosophila endogenous small RNAs bind to Argonaute2 in somatic cells. Nature 2008, 453:793-797.

15. Lu J, Shen Y, Wu Q, Kumar S, He B, Shi S, Carthew RW, Wang SM, Wu C-I: The birth and death of microRNA genes in Drosophila. Nat Genet 2008, 40:351-355.

16. Okamura K, Chung W-J, Ruby JG, Guo H, Bartel DP, Lai EC: The Drosophila hairpin RNA pathway generates endogenous short interfering RNAs. Nature 2008, 453:803-806.

17. Ruby JG, Stark A, Johnston WK, Kellis M, Bartel DP, Lai EC: Evolution, biogenesis, expression, and target predictions of a substantially expanded set of Drosophila microRNAs. Genome Res 2007, 17:1850-1864.

18. Seitz H, Ghildiyal M, Zamore PD: Argonaute loading improves the $5^{\prime}$ precision of both microRNAs and their miRNA* strands in flies. Current Biol 2008, 18:147-151.

19. Tyler DM, Okamura K, Chung W-J, Hagen JW, Berezikov E, Hannon GJ, Lai EC: Functionally distinct regulatory RNAs generated by bidirectional transcription and processing of microRNA loci. Genes Dev 2008, 22:26-36

20. Yin $\mathrm{H}$, Lin $\mathrm{H}$ : An epigenetic activation role of Piwi and a Piwi-associated piRNA in Drosophila melanogaster. Nature 2007, 450:304-308.

21. Kawaji H, Nakamura M, Takahashi Y, Sandelin A, Katayama S, Fukuda S, Daub C, Kai C, Kawai J, Yasuda J, et al: Hidden layers of human small RNAs. BMC Genomics 2008, 9:157

22. Taft RJ, Glazov EA, Lassmann T, Hayashizaki Y, Carninci P, Mattick JS: Small RNAs derived from snoRNAs. RNA 2009, 15:1233-1240.

23. Ender $C$, Krek $A$, Friedländer MR, Beitzinger $M$, Weinmann $L$, Chen $W$, Pfeffer S, Rajewsky N, Meister G: A human snoRNA with microRNA-like functions. Mol Cell 2008, 32:519-528.

24. Chen H-M, Wu S-H: Mining small RNA sequencing data: a new approach to identify small nucleolar RNAs in Arabidopsis. Nucl Acids Res 2009, 37: e69.

25. Griffiths-Jones S, Saini HK, van Dongen S, Enright AJ: miRBase: tools for microRNA genomics. Nucl Acids Res 2008, 36:D154-158.

26. Drysdale R: FlyBase: a database for the Drosophila research community. Methods Mol Biol 2008, 420:45-59.

27. Yuan G, Klambt C, Bachellerie J-P, Brosius J, Huttenhofer A: RNomics in Drosophila melanogaster: identification of 66 candidates for novel nonmessenger RNAs. Nucl Acids Res 2003, 31:2495-2507.

28. Mörl M, Marchfelder A: The final cut: The importance of tRNA 3'processing. EMBO Rep 2001, 2:17

29. Liang $Y$, Ridzon D, Wong $L$, Chen $C$ : Characterization of microRNA expression profiles in normal human tissues. BMC Genomics 2007, 8:166.

30. Bachellerie J-P, Cavaille J, Huttenhofer A: The expanding snoRNA world. Biochimie 2002, 84:775-790

31. Balakin AG, Smith L, Fournier MJ: The RNA world of the nucleolus: Two major families of small RNAs defined by different box elements with related functions. Cell 1996, 86:823-834.

32. Altschul SF, Madden TL, Schaffer AA, Zhang J, Zhang Z, Miller W, Lipman DJ: Gapped BLAST and PSI-BLAST: a new generation of protein database search programs. Nucl Acids Res 1997, 25:3389-3402.

33. Mount SM, Gotea V, Lin CF, Hernandez K, Makalowski W: Spliceosomal small nuclear RNA genes in 11 insect genomes. RNA 2007, 13:5-14.
34. Kiss-László Z, Henry Y, Bachellerie J-P, Caizergues-Ferrer M, Kiss T: Sitespecific ribose methylation of preribosomal RNA: A novel function for small nucleolar RNAs. Cell 1996, 85:1077-1088.

35. Schneider I: Cell lines derived from late embryonic stages of Drosophila melanogaster. J Embryol Exp Morphol 1972, 27:353-365.

36. Chan PP, Lowe TM: GtRNAdb: a database of transfer RNA genes detected in genomic sequence. Nucl Acids Res 2009, 37:D93-97.

37. Ullu E, Tschudi C: Alu sequences are processed 7SL RNA genes. Nature 1984, 312:171-172

38. Siepel A, Bejerano G, Pedersen JS, Hinrichs AS, Hou M, Rosenbloom K, Clawson H, Spieth J, Hillier LW, Richards S, et al: Evolutionarily conserved elements in vertebrate, insect, worm, and yeast genomes. Genome Res 2005, 15:1034-1050.

39. Gruber AR, Kilgus C, Mosig A, Hofacker IL, Hennig W, Stadler PF: Arthropod 7SK RNA. Mol Biol Evol 2008, 25:1923-1930.

40. Hertel J, Hofacker IL, Stadler PF: SnoReport: computational identification of snoRNAs with unknown targets. Bioinformatics 2008, 24:158-164.

41. Pang KC, Frith MC, Mattick JS: Rapid evolution of noncoding RNAs: lack of conservation does not mean lack of function. Trends Genet 2006, 22:1-5.

42. Malone CD, Brennecke J, Dus M, Stark A, McCombie WR, Sachidanandam R, Hannon GJ: Specialized piRNA pathways act in germline and somatic tissues of the Drosophila ovary. Cell 2009, 137:522-535.

43. Smit A, Hubley R, Green P: RepeatMasker Open-3.0 http://www. repeatmasker.org. 1996.

44. Kuhn RM, Karolchik D, Zweig AS, Trumbower H, Thomas DJ, Thakkapallayil A, Sugnet CW, Stanke M, Smith KE, Siepel A, et al: The UCSC genome browser database: update 2007. Nucl Acids Res 2007, 35 : D668-673.

45. Boyle El, Weng S, Gollub J, Jin H, Botstein D, Cherry JM, Sherlock G: GO: TermFinder-open source software for accessing Gene Ontology information and finding significantly enriched Gene Ontology terms associated with a list of genes. Bioinformatics 2004, 20:3710-3715.

46. Pall GS, Hamilton AJ: Improved northern blot method for enhanced detection of small RNA. Nat Protocols 2008, 3:1077-1084.

47. Hofacker IL: Vienna RNA secondary structure server. Nucl Acids Res 2003, 31:3429-3431.

doi:10.1186/1471-2164-11-77

Cite this article as: Jung et al:: Identification of novel non-coding RNAs using profiles of short sequence reads from next generation sequencing data. BMC Genomics 2010 11:77.

\section{Submit your next manuscript to BioMed Central and take full advantage of:}

- Convenient online submission

- Thorough peer review

- No space constraints or color figure charges

- Immediate publication on acceptance

- Inclusion in PubMed, CAS, Scopus and Google Scholar

- Research which is freely available for redistribution

Submit your manuscript at www.biomedcentral.com/submit
C Biomed Central 